\title{
A monitorização residencial da pressão arterial e o controle de um grupo de hipertensos*
}

\author{
HOME BLOOD PRESSURE MONITORING AND CONTROL IN A GROUP OF \\ HYPERTENSIVE PATIENTS
}

\begin{abstract}
MONITOREO RESIDENCIAL DE LA PRESIÓN ARTERIAL Y CONTROL DE UN GRUPO DE HIPERTENSOS
\end{abstract}

\section{Giane Christina Alves da Silva ${ }^{1}$, Angela Maria Geraldo Pierin²}

\section{RESUMO}

Comparar a medida de consultório com a monitorização residencial da pressão arterial (MRPA), avaliar o controle da pressão e caracterizar o efeito do avental branco. Pesquisa de campo, quantitativa com 71 hipertensos. A medida da pressão em consultório foi feita pela enfermeira. A monitorização residencial da pressão arterial foi realizada durante 7 dias. 0 efeito do avental branco foi quantificado para diferenças entre a medida de consultório e a monitorização residencial da pressão arterial nas faixas 1 a 5, 6 a 10 e $>10 \mathrm{mmHg}$. A medida da pressão de consultório foi significativamente maior $(p<0,05)$ do que a monitorização residencial da pressão arterial. O controle da pressão foi 9,9\% na medida de consultório e 23,9\% na MRPA. O efeito do avental branco $>10 \mathrm{mmHg}$ para a sistólica foi $57,7 \%$ e para a diastólica, 32,4\%, na faixa de 6 a $10 \mathrm{mmHg}$. A medida da pressão em casa avaliou melhor o controle dos hipertensos.

\section{DESCRITORES}

Hipertensão

Medida

Monitoramento

Hipertensão do jaleco branco

Controle

\begin{abstract}
This qualitative study was performed with 71 hypertensive patients, with the objectives to compare outpatient and home blood pressure monitoring (HBPM), to assess blood pressure control, and characterize white-coat hypertension. A nurse performed the outpatient blood pressure measurement. The home blood pressure monitoring was carried out over seven days. White-coat hypertension was quantified as a difference between the outpatient measurement and home blood pressure monitoring in the ranges from 1 to 5,6 to 10 and $>10 \mathrm{mmHg}$. The outpatient blood pressure measurement was significantly higher $(p<0.05)$ than the home blood pressure measurement. Pressure control corresponded to $9.9 \%$ in the outpatient measurement and $23.9 \%$ in the home blood pressure measurement. The white-coat effect $>10 \mathrm{mmHg}$ was $57.7 \%$ for systolic and $32.4 \%$ for diastolic pressure, in the range from 6 to $10 \mathrm{mmHg}$. Home blood pressure measurement provided a better assessment of hypertensive patients' control.
\end{abstract}

\section{DESCRIPTORS}

Hypertension

Measures

Monitoring

White coat hypertension

Control

\begin{abstract}
RESUMEN
Comparación de la medición en consultorio con el monitoreo residencial de la presión arterial (MRPA), evaluación del control de presión y caracterización del efecto del delantal blanco. Investigación de campo, cuantitativa, con 71 hipertensos. La medición de presión en consultorio fue realizada por la enfermera. EI MRPA se efectuó durante siete días. El efecto del delantal blanco se cuantificó para diferencias entre la medición de consultorio y el MRPA en las fajas 1-5, 6-10 y >10mmHg. La medición de presión en consultorio fue significativamente mayor $(p<0,05)$ que el monitoreo residencial de presión arterial. La presión se mantuvo bajo control en el $9,9 \%$ de la medición de consultorio y $23,9 \%$ de la MRPA. El efecto del delantal blanco $>10$ $\mathrm{mmHg}$ para la sistólica fue de $57,7 \%$ y para la diastólica, 32,4\%, en la faja 6-10 mmHg. La medición de presión domiciliaria evaluó mejor el control de los hipertensos.
\end{abstract}

\footnotetext{
* Extraído da dissertação "Uso da medida da pressão em casa como estratégia de controle da pressão arterial de um grupo de hipertensos de Peruíbe, SP", Programa de Enfermagem na Saúde do Adulto da Escola de Enfermagem da Universidade de São Paulo, 2010. ${ }^{1}$ Mestre em Enfermagem pelo Programa de Pós Graduação em Enfermagem na Saúde do Adulto da Escola de Enfermagem da Universidade de São Paulo. São Paulo, SP, Brasil. giane.alves@hotmail.com ${ }^{2}$ Professora Titular da Escola de Enfermagem da Universidade de São Paulo. São Paulo, SP, Brasil. pierin@usp.br
} 


\section{INTRODUÇÃO}

A hipertensão arterial sistêmica é uma condição clínica multifatorial caracterizada basicamente por níveis elevados da pressão arterial|(1). Por ser definida pela elevação dos níveis da pressão arterial, a sua mensuração é um recurso imprescindível para a delimitação entre a normotensão e a hipertensão. A alta prevalência da hipertensão arterial e as baixas taxas de controle conferem ao procedimento de medida da pressão arterial importante papel tanto na detecção como no manejo da hipertensão.

Quanto à prevalência de hipertensão em nosso meio, não existe estudo de magnitude nacional, e sim dados isolados e regionais. De um modo geral estima-se que $30 \%$ da população adulta seja hipertensa. A hipertensão é a condição crônica mais freqüente nas faixas etárias superiores e está intimamente associada à doença cerebrovascular e isquêmica do coração, que correspondem às doenças mais prevalentes do aparelho circulatório, principal causa de morte entre idosos brasileiros. Em 2006 as doenças cerebrovasculares, isquêmicas do coração e hipertensão arterial foram responsáveis por $9,4 \%, 8,8 \%$ e $3,6 \%$ dos óbitos por doenças do aparelho circulatório respectivamente, o que corresponde a 223.800 mortes, $74 \%$ dos óbitos por doença cardiovascular(2).

A medida da pressão arterial é um elemento crucial para o estabelecimento do diagnóstico de hipertensão arterial e avaliação da eficácia do tratamento, devendo ser realizada em toda avaliação de saúde por profissionais adequadamente treinados ${ }^{(1)}$.

Apesar de ser um procedimento simples e de fácil realização, a medida da pressão arterial, está sujeita a erros que podem estar relacionados ao equipamento, técnica, ambiente, paciente ou observador. Em relação ao paciente, os possíveis erros podem estar relacionados à medida após a atividade física, comer, fumar, ingestão de bebida alcoólica ou cafeína antes da medida, posição incorreta ou a posição do braço. Estudos ${ }^{(3-4)}$ realizados em nosso meio, têm mostrado fragilidades no conhecimento da equipe de enfermagem que envolve a assistência aos hipertensos. Os erros relacionados ao observador estão ligados a posição incorreta dos olhos, ao arredondamento dos valores para dígitos terminados em zero ou cinco, a pressão excessiva do estetoscópio deformando a artéria, inflar excessivamente o manguito provocando dor ou deflacionar rapidamente, mãos e equipamentos gelados, identificação incorreta dos sons sistólico e diastólico e a interação incorreta com o paciente. Para uma aferição fidedigna, o equipamento deverá estar calibrado, a relação entre o tamanho do manguito e o braço do paciente deverá estar adequada, caso contrário, na utilização de um manguito inadequado, pode acarretar valores subestimados ou superestimados da pressão arterial. A largura da bolsa inflá- vel deverá corresponder a $40 \%$ da circunferência do braço e o seu comprimento deverá envolver $80 \%$ do braço, mantendo uma relação de 1:2.

Na medida da pressão arterial a interação entre o paciente e o observador e ambiente são fatores que interferem nos valores obtidos. Segundo a IV Diretriz para uso da monitorização ambulatorial da pressão arterial e II Diretriz para uso da monitorização residencial da pressão arterial, a medida fora do consultório pode afastar o efeito do avental branco na medida da pressão arterial(5). Estudo realizado em Unidades Básicas de Saúde comparando a média dos quatro dias de medida residencial e a medida realizada pela enfermeira mostrou que não houve diferença para a pressão sistólica, porém a pressão diastólica residencial foi significativamente menor que a registrada pela enfermeira caracterizando o efeito do avental bran$\mathrm{co}^{(6)}$. Também em nosso meio, outra investigação mostrou que a medida em casa, além de mostrar valores menores do que a medida de consultório, apresentou melhor valor prognóstico na identificação de lesão de órgãos alvo, como a hipertrofia de ventrículo esquerdo ${ }^{(7)}$.

De acordo com as VI Diretrizes Brasileiras de Hipertensão(5) a medida da pressão arterial de consultório ainda é o método usual para o diagnóstico e tratamento da hipertensão, porém indica a medida fora do consultório, como uma possibilidade de elucidação do diagnóstico por afastar a hipertensão do avental branco.

Após o desenvolvimento de aparelhos automáticos, a medida realizada fora do consultório médico passou a ser estudada com maior propriedade. Estes aparelhos além de proporcionar e armazenar grande número de medidas em situações fora do consultório médico independem da habilidade do paciente de medir a própria pressão arterial. A monitorização residencial da pressão arterial (MRPA) é o registro da pressão arterial por método indireto, realizado pelo paciente ou outra pessoa treinada, durante a vigília, no domicílio ou no trabalho. A identificação do hipertenso do avental branco, a presença do efeito do avental branco, a hipertensão mascarada e a avaliação da terapêutica anti-hipertensiva estão dentre as suas principais indicações ${ }^{(1)}$. A monitorização residencial da pressão arterial é distinta da auto medida, que se trata de medidas isoladas e de forma não sistematizada, realizadas pelo paciente em seu domicílio.

Estudos apontam a monitorização residencial da pressão arterial como um método mais apropriado do que a medida de consultório e tão boa quanto à monitorização ambulatorial da pressão arterial, pois promove diagnóstico preciso e melhor acompanhamento da hipertensão ao longo do tratamento ${ }^{(8-9)}$. Investigação realizada em um ambulatório de um Hospital de Ensino na cidade 
de São Paulo, evidenciou o efeito benéfico da monitorização residencial da pressão arterial na avaliação do controle dos hipertensos, superando inclusive a avaliação da monitorização ambulatorial da pressão arterial (MAPA). Os valores obtidos com a monitorização ambulatorial da pressão arterial e monitorização residencial da pressão arterial foram menores que os obtidos no consultório, o que confirma achados na literatura de que a pressão arterial de consultório é mais elevada do que as obtidas nos métodos $\operatorname{citados}^{(10)}$.

Portanto, o presente estudo teve como objetivos: 1 - Comparar a medida realizada no consultório com a monitorização residencial da pressão arterial. 2 - Avaliar o controle da pressão arterial de acordo com a medida realizada em consultório e MRPA. 3 - Caracterizar o efeito do avental branco.

\section{MÉTODO}

Trata-se de estudo descritivo com abordagem quantitativa. Foram estudados 71 adultos portadores de hipertensão arterial essencial, usuários de um Centro de Referência em Hipertensão e Diabetes da cidade de Peruíbe, situada no litoral paulista. Os critérios de inclusão dos hipertensos foram: a) concordância do paciente para a participação no estudo, através da assinatura do termo de consentimento livre e esclarecido; $b$ ) ser hipertenso essencial estágio I ou II, atendido há mais de 6 meses na unidade; d) ter idade igual ou superior a 18 anos; e d) pertencer à comunidade de abrangência do município de Peruíbe. Os critérios de exclusão foram: a) hipertensas grávidas; b) hipertensos com história de abuso de drogas ou distúrbios mentais que possam invalidar o consentimento livre e esclarecido; c) hipertensão arterial secundária; e d) Diabetes tipo I. O estudo foi aprovado pelo Comitê de Ética da Escola de Enfermagem da Universidade de São Paulo.

A medida da pressão arterial de consultório foi realizada pela enfermeira com aparelho automático validado (OMROM HEM 705 CP), na unidade de saúde em ambiente calmo, sem ruídos; na posição sentada, após 5- 10 minutos de repouso; com o membro superior esquerdo apoiado na altura do coração; três vezes consecutivas, sendo o intervalo entre uma e outra medida de 1 a 2 minutos; com manguito adequado ao tamanho do braço; sem ter praticado atividade física 60-90 minutos; com a bexiga vazia; e sem ter realizado refeição, ingerido bebida alcoólica ou cafeinada pelo menos há 30 minutos. Foram considerados anormais valores acima de $140 / 90 \mathrm{mmHg}^{(1)}$ usados para caracterizar o controle dos hipertensos.

Para a monitorização residencial da pressão arterial (MRPA) os hipertensos foram orientados pela enfermeira pesquisadora na unidade de saúde sobre a forma cor- reta do uso do aparelho de medida da pressão, impressão e anotação dos valores da pressão em formulário próprio. A monitorização residencial da pressão arterial foi realizada durante sete dias consecutivos, três vezes pela manhã e três vezes à noite, no período compreendido entre $06 \mathrm{~h} 00-10 \mathrm{~h} 00$ e $18 \mathrm{~h} 00-22 \mathrm{~h} 00$, respectivamente. As orientações ao paciente para a realização da monitorização residencial da pressão arterial foram as seguintes: realizar medidas em ambiente tranquilo, com temperatura agradável, sem estar com bexiga cheia, sem ter praticado exercícios físicos há 60 minutos, sem ter ingerido bebidas alcoólicas, café, alimentos, ou fumado até 30 minutos antes, e sem falar durante as medidas; efetuar a medida na posição sentada após 5 minutos de repouso, com as costas apoiadas, pernas descruzadas e pés apoiados no chão, com intervalo entre as medidas de 1 minuto; utilizar sempre o braço esquerdo apoiado na altura do coração, com a palma da mão voltada para cima e sem movimentação durante as medidas; colocar o manguito no braço livre de roupas e sem garroteamento por roupas apertadas.

Valores acima de 135/85 mmHg da monitorização residencial da pressão arterial foram considerados alte$\operatorname{rados}^{(5)}$ na avaliação do controle. Em relação à qualidade do procedimento, foram aceitas as medidas válidas em todos os dias e excluídas medidas aberrantes tais como diastólica acima de $140 \mathrm{mmHg}$ e abaixo de $40 \mathrm{mmHg}$, sistólica abaixo de $70 \mathrm{mmHg}$ e acima de $250 \mathrm{mmHg}$ e pressão de pulso menor que $20 \mathrm{mmHg}$ ou maior que $100 \mathrm{mmHg}$, desde que não existisse justificativa clínica. Em ambas as modalidades de aferição foram realizadas três medidas e o valor usado foi obtido pela média da segunda e da terceira medida. Para coleta dos dados foi utilizada uma ficha de identificação com as variáveis sócio demográficas dos participantes.

O efeito do avental branco foi caracterizado pela diferença entre a medida da pressão arterial de consultório e a monitorização residencial da pressão arterial, estratificada nas seguintes faixas: $<0,1-5,6-10$ e $>10 \mathrm{mmHg}$. $\mathrm{Na}$ análise dos dados as variáveis classificatórias são apresentadas descritivamente com frequências absolutas (n) e percentagens (\%). As variáveis quantitativas com distribuição normal são apresentadas descritivamente com médias e desvio padrão. As variáveis contínuas foram analisadas com uso de análise de perfis. Os valores de $p<0,05$ foram considerados estatisticamente significantes.

\section{RESULTADOS}

A caracterização da amostra estudada evidenciou o predomínio de hipertensos na faixa etária acima de 60 anos, do sexo masculino (53,5\%), brancos $(77,5 \%)$, com companheiro $(76,1 \%)$, com ensino fundamental completo $(51,4 \%)$, aposentados $(53,5 \%)$ e com tempo de tratamento na unidade em torno de 5 anos (Tabela 1). 
Tabela 1 - Características biossociais dos hipertensos estudados - Peruíbe, SP, 2010

\begin{tabular}{|c|c|c|}
\hline Variáveis & $\mathbf{N}$ & $\%$ \\
\hline \multicolumn{3}{|l|}{ Sexo } \\
\hline Feminino & 33 & 46,5 \\
\hline Masculino & 38 & 53,5 \\
\hline \multicolumn{3}{|l|}{ Etnia } \\
\hline Branca & 55 & 77,5 \\
\hline Negra & 5 & 7,0 \\
\hline Mulata & 9 & 12,7 \\
\hline Amarela & 2 & 2,8 \\
\hline \multicolumn{3}{|l|}{ Estado civil } \\
\hline Solteiro & 3 & 4,2 \\
\hline Casado & 44 & 62,0 \\
\hline Separado & 5 & 7,0 \\
\hline Amasiado & 10 & 14,1 \\
\hline Viúvo & 9 & 12,7 \\
\hline \multicolumn{3}{|l|}{ Escolaridade } \\
\hline Analfabeto/ Lê e escreve & 7 & 9,9 \\
\hline Fundamental & 36 & 50,7 \\
\hline Médio & 18 & 25,3 \\
\hline Superior & 9 & 14,1 \\
\hline \multicolumn{3}{|l|}{ Ocupação } \\
\hline Aposentado & 38 & 53,5 \\
\hline Manual não especializado & 21 & 29,6 \\
\hline Manual especializado & 20 & 14,1 \\
\hline Não manual & 2 & 2,8 \\
\hline Idade (média \pm Desvio Pac & & $63,3 \pm 11,0$ \\
\hline Tempo de tratamento $(\mathrm{m}$ & anos) & $5,11 \pm 3,53$ \\
\hline
\end{tabular}

A avaliação da medida da pressão arterial mostrou que os valores obtidos na unidade de atendimento dos hipertensos foram significativamente mais elevados $(p<0,05)$ do que os da monitorização residencial da pressão arterial $(157,7 \pm 12,3 / 91,4 \pm 8,2$ vs $145,1 \pm 16,2 / 85,0 \pm 9,4$ $\mathrm{mmHg}$ ) (Figura 1).

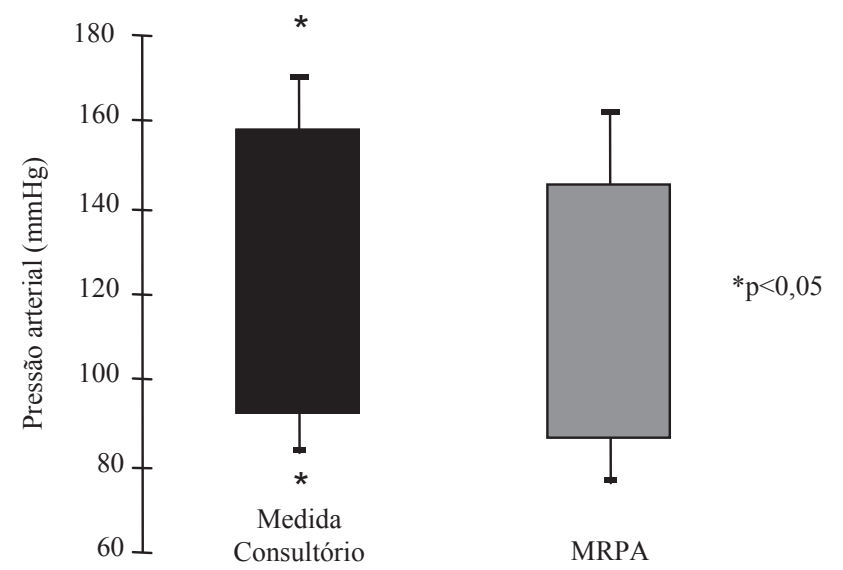

Figura 1 - Medida da pressão arterial dos hipertensos estudados no consultório e na monitorização residencial da pressão arterial (MRPA) - Peruíbe, SP, 2010

A monitorização residencial da pressão arterial evidenciou valores significativamente maiores $(p<0,05)$ para a pressão sistólica e diastólica nos períodos da manhã e da noite na comparação do primeiro com os demais dias da semana. Os valores dos dias 2, 3 e 4 não foram distintos, enquanto que no dia 5 , houve decréscimo estatisticamente significante $(p<0,05)$, com exceção apenas para a pressão diastólica noturna. Embora os níveis tensionais sistólicos tenham aumentado no sexto e sétimo dia, ainda assim foram menores em relação aos obtidos no início da semana (Tabela 2).

Tabela 2 - Monitorização residencial da pressão arterial (MRPA) dos hipertensos estudados, nos períodos manhã e noite, comparação entre os sete dias da semana - Peruíbe, SP, 2010

\begin{tabular}{|c|c|c|c|c|}
\hline \multirow[b]{2}{*}{ Dias } & \multicolumn{2}{|c|}{$\begin{array}{c}\text { MRPA Sistólica } \\
\text { (Média } \pm \text { Desvio Padrão, } \\
\text { mmHg) }\end{array}$} & \multicolumn{2}{|c|}{$\begin{array}{c}\text { MRPA Diastólica } \\
\text { (Média } \pm \text { Desvio Padrão } \\
\text { mmHg) }\end{array}$} \\
\hline & Manhã & Noite & Manhã & Noite \\
\hline 1 & $150,8 \pm 17,2 *$ & $151,4 \pm 21,2 *$ & $90,2 \pm 10,9^{*}$ & $87,5 \pm 11,6^{*}$ \\
\hline 2 & $144,2 \pm 14,8$ & $146,6 \pm 20,4$ & $87,0 \pm 10,2$ & $84,6 \pm 11,4$ \\
\hline 3 & $144,7 \pm 18,5$ & $146,2 \pm 20,8$ & $86,3 \pm 10,5$ & $84,4 \pm 12,3$ \\
\hline 4 & $145,4 \pm 18,1$ & $144,1 \pm 19,8$ & $86,4 \pm 11,0$ & $83,7 \pm 11,1$ \\
\hline 5 & $140,7 \pm 17,3 \dagger$ & $142,3 \pm 20,5 \S$ & $84,3 \pm 10,6 \S$ & $83,0 \pm 11,6$ \\
\hline 6 & $144,0 \pm 18,3$ & $144,2 \pm 21,9$ & $85,5 \pm 10,9$ & $83,9 \pm 11,0$ \\
\hline 7 & $144,6 \pm 19,0$ & $143,3 \pm 20,2$ & $85,9 \pm 10,3$ & $83,6 \pm 10,5$ \\
\hline Média & \multicolumn{2}{|c|}{$145,1 \pm 16,2$} & \multicolumn{2}{|c|}{$85,0 \pm 9,4$} \\
\hline
\end{tabular}

O efeito do avental branco avaliado pela diferença entre a medida da pressão arterial no consultório do ambulatório e a medida realizada em casa (MRPA) mostrou que mais da metade $(57,7 \%)$ dos hipertensos apresentou magnitude do efeito acima de $10 \mathrm{mmHg}$ para a sistólica, enquanto que para a pressão diastólica o efeito foi maior na faixa de 6 a 10 $\mathrm{mmHg}(32,4 \%)$. Cerca de $80 \%$ dos hipertensos apresentaram efeito do avental branco. Destaca-se ainda, que houve efeito contrário em 16,9\% e 19,7\% dos hipertensos, para as pressões sistólica e diastólica, respectivamente.

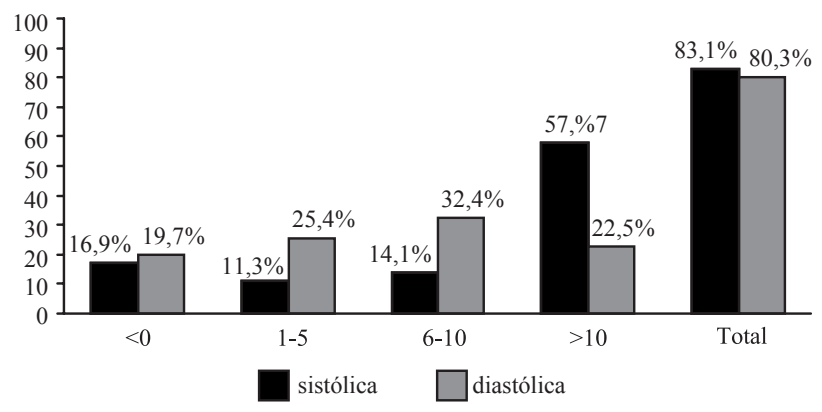

Diferenças entre a medida de consultório e MRPA $(\mathrm{mmHg})$

Figura 2 - Percentual de hipertensos de acordo com o efeito do avental branco para as pressões sistólica e diastólica - Peruíbe, SP, 2010 
A avaliação do controle da pressão arterial mostrou que de acordo com a medida de consultório, apenas 9,9\% dos hipertensos estudados estavam com seus níveis tensionais controlados (<140/90 mmHg). Por outro lado, considerando a medida realizada em casa, o percentual de controle $(<135 / 85 \mathrm{mmHg}$ ) foi mais que o dobro $(23,9 \%)$ (Figura 3$)$.
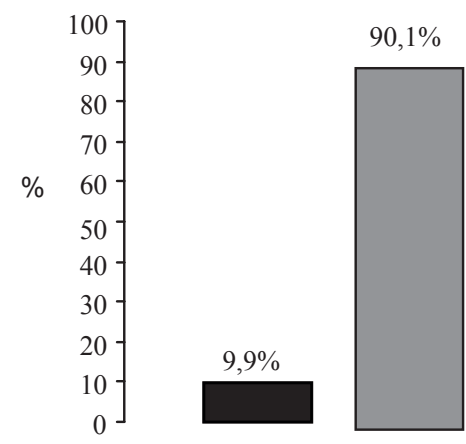

Medida de Consultório

$\%$ Hipertensos controlados

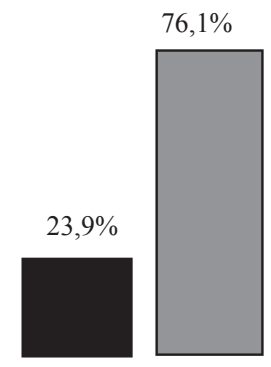

MRPA

$\%$ Hipertensos não controlados
Figura 3 - Percentual de hipertensos controlados e não controlados de acordo com a medida de consultório e monitorização residencial da pressão arterial (MRPA) - Peruíbe, SP, 2010

\section{DISCUSSÃO}

O principal achado do presente estudo mostrou que a medida da pressão arterial realizada fora do local de atendimento do hipertenso, apresentou níveis significativamente mais baixos, revelando mais uma vez a importância da medida domiciliar. A influência do observador na medida da pressão arterial é um fato amplamente estudado na literatura. Estudo pioneiro nessa área realizado há mais de duas décadas mostrou que as presenças do médico e da enfermeira provocaram elevação na pressão arterial avaliada pelo método direto, porém a elevação provocada pela presença da enfermeira foi menos acentuada do que a do médico. Os autores concluem que repetidas visitas do médico em pequenos espaços de tempo não evitaram a superestimação na pressão arterial, mas pode ser reduzida pela medida feita pela enfermeira ${ }^{(11)}$. Estudo mais recente também mostrou medidas realizadas pela enfermeira significativamente menores que as do médico ${ }^{(12)}$. Metanálise ${ }^{(13)}$ sobre $o$ assunto evidenciou aumentos iguais ou superiores a $30 \mathrm{mmHg}$ quando a pressão arterial foi aferida por médico. Nessa mesma linha, estudo realizado em nosso meio, analisou a medida da pressão arterial realizada pelo paciente, pela enfermeira, pelo médico, pela monitorização ambulatorial e monitorização residencial da pressão arterial, e os resultados mostraram que os valores maiores foram obtidos pela aferição do médico ${ }^{(14)}$. Reforçando a importância do papel do observador na medida da pressão arterial outra investigação ${ }^{(15)}$ comparou a medida da pressão realizada pela enfermeira e pelo paciente evidenciando que os valores obtidos pela enfer- meira foram menores e que estas medidas mesmo sendo repetidas após cinco minutos, embora a pressão tenha diminuído, ainda assim os valores obtidos pela enfermeira foram menores. Em nosso meio, estudo também comparou a medida da pressão entre diferentes observadores e verificou-se que as realizadas pelo paciente e enfermeira foram próximas ${ }^{(16)}$.

As diferenças entre as medida da pressão realizadas no consultório e em casa ou pela monitorização ambulatorial (MAPA), caracterizam o fenômeno do avental branco que compreende diferentes situações. A hipertensão do avental branco é quando há hipertensão na medida da pressão arterial no consultório, e normotensão pela monitorização ambulatorial da pressão arterial ou medida domiciliar. A hipertensão mascarada é o inverso da hipertensão do avental branco. Outra possibilidade é o efeito do avental branco que se caracteriza pela elevação dos níveis pressóricos quando a medida da pressão é realizada pelo médico no consultório quando comparados com os registrados pela monitorização ambulatorial da pressão arterial (MAPA) ou na medida domiciliar, independentemente do diagnóstico de normotensão ou hipertensão. Deste modo, no efeito do avental branco não há mudança no diagnóstico do paciente. Em nosso meio, ainda são escassos investigações sobre esse assunto. Estudo realizado em uma liga de hipertensão de um hospital escola comparou registros de 670 exames de Monitorização ambulatorial da pressão com a medida de consultório e os resultados mostraram que $46 \%$ eram hipertensos, $12 \%$ com hipertensão mascarada, $20 \%$ hipertensos do avental branco e $27 \%$ apresentaram efeito do avental branco ${ }^{(17)}$.

Foi observado no presente estudo, que a medida realizada no consultório, apesar de ter sido realizada pela enfermeira, ainda apresentou valores significativamente mais elevados quando comparada às medidas realizadas em casa pelo paciente na monitorização residencial da pressão arterial (MRPA). Dessa forma, mesmo que a influência do observador tenha sido amenizada, a influência do ambiente ainda permaneceu. Tal fato repercutiu no controle dos níveis da pressão arterial, apenas $10 \%$ da dos hipertensos estudados na medida de consultório, apresentavam a pressão arterial controlada.

A preocupação em medir a pressão arterial fora do consultório não é recente. Relato da literatura datado de $1940^{(18)}$ comparou registro de pressão arterial de hipertensos em casa e no consultório e relataram, pela primeira vez, o fenômeno da hipertensão do avental branco, ao evidenciar que os registros no domicílio foram inferiores aos obtidos no consultório.

O diagnóstico da hipertensão do avental branco efeito do avental branco é um grande desafio aos profissionais de saúde, pois o método da medida da pressão arterial no consultório ainda é o mais utilizado para o diagnóstico da pressão arterial. Embora no presente estudo, a medida de consultório tenha sido realizada pela enfermeira, a moni- 
torização residencial da pressão arterial (MRPA) apresentou diminuição dos níveis pressóricos, reforçando mais uma vez a importância dessa estratégia de avaliação da pressão arterial, não só na confirmação do diagnóstico da hipertensão, mas também no manejo do tratamento anti-hipertensivo. Tal fato pode ser evidenciado quando se verifica que o controle dos hipertensos foi extremamente distinto quando comparadas as duas formas de avaliação da pressão arterial. Mais da metade dos hipertensos estudados apresentaram efeito do avental branco maior que $10 \mathrm{mmHg}$ na pressão sistólica o que pode implicar em super tratamento dessas pessoas, com aumento no uso de medicamentos anti-hipertensivos.

Apesar do controle dos hipertensos se revelar maior pela avaliação da pressão em casa, observa-se que os índices ainda estão muito baixos. Em nosso meio, tem-se verificado que apenas cerca de $30 \%$ dos hipertensos estão controlados. Dessa forma, estudar alternativas que possam mudar esse panorama torna-se tão importante e a medida da pressão em casa é uma possibilidade. Estudos têm evidenciado que a realização da medida em casa pode promover maior adesão ao tratamento e consequentemente maior controle dos níveis da pressão arterial|(19-20).

No presente estudo não foram encontradas diferenças entre as médias pressóricas dos períodos da manhã e noite tanto para a sistólica como para diastólica. Dados similares ao de outro estudo, também realizado em nosso meio, com avaliação da pressão em casa durante sete dias, no qual inclusive as médias das pressões sistólica e diastólica foram avaliadas em três períodos, matutino, vespertino e noturno(7). Publicação nacional de revisão

\section{REFERÊNCIAS}

1. Sociedade Brasileira Cardiologia; Sociedade Brasileira de Hipertensão; Sociedade Brasileira de Nefrologia. VI Diretrizes Brasileiras de Hipertensão Arterial. Rev Bras Hipertens. 2010;17(1):4-60.

2. Brasil. Ministério da Saúde; Secretaria de Vigilância em Saúde, Departamento de Análise de Situação de Saúde. Saúde Brasil 2008: 20 anos de Sistema Único de Saúde (SUS) no Brasil. Brasília; 2009.

3. Silva SSBE, Colósimo FC, Pierin AMG. The effect of educational interventions on nursing team knowledge about arterial hypertension. Rev Esc Enferm USP [Internet]. 2010 [cited 2011 Mar 17];44(2):488-96. Available from: http://www.scielo.br/pdf/reeusp/v44n2/en_36.pdf

4. Rabello CCP, Pierin AMG, Mion Junior D. O conhecimento de profissionais da área da saúde sobre a medida da pressão arterial. Rev Esc Enferm USP. 2004;38(2):127-34. destacou a importância no manejo dos hipertensos utilizando a monitorização residencial da pressão arterial. As autoras ressaltam que se trata de método que permite a avaliação do comportamento da pressão arterial a médio e longo prazo, sendo eficaz na avaliação da terapêutica anti-hipertensiva, além de promover condições que favorecem a adesão ao tratamento e consequentemente elevar os índices de controle da doença( ${ }^{(21)}$.

\section{CONCLUSÃO}

Os dados do presente estudo reforçam mais uma vez que a medida da pressão arterial sofre influência do local em que é realizada e que a medida em casa contribuiu não só para identificação do efeito do avental branco, observado na maioria dos hipertensos, mas também proporcionou avaliação do real controle da pressão arterial. A avaliação efetiva do controle da pressão é de suma importância no manejo do tratamento dos hipertensos. A manutenção de níveis pressóricos elevados pode acarretar complicações com danos em órgãos alvo com sérias repercussões para a pessoa hipertensa. Outro fato evidenciado foi o baixo índice de controle dos hipertensos estudados. Mesmo quando avaliados pela medida residencial, os índices obtidos estão muito aquém dos desejados. Essa é uma tarefa que merece atenção por parte de todos que atuam junto aos hipertensos e a avaliação da pressão arterial em casa pelo paciente pode ser um recurso pela possibilidade de maior envolvimento com sua problemática de saúde. Diante da incipiência de dados sobre a questão estudada, o nosso trabalho fornece dados importantes para futuras comparações.

5. Alessi A, Brandão AA, Pierin A, Feitosa AM, Machado CA, Forjaz CLM, et al. IV Guideline for ambulatory blood pressure monitoring. II Guideline for home blood pressure monitoring. IV ABPM/II HBPM. Arq Bras Cardiol. 2005;85(2):1-18.

6. Pierin AMG, Alavarce DC, Santos GAS. Medida residencial da pressão arterial em hipertensos atendidos em Unidades Básicas de Saúde. Hipertensão. 2006;9(2):60-4.

7. Gomes MAM, Pierin AMG, Segre CA, Mion Junior D. Monitorização residencial da pressão arterial e monitorização ambulatorial da pressão arterial versus medida de pressão arterial no consultório. Arq Bras Cardiol. 1998;71(4):581-5.

8. Cappucio FP, Kerry SM, Forbes L, Donald A. Blood pressure control by home monitoring: meta-analysis of randomized trails. BMJ. 2004;329(7458):145-9. 
9. Stergiou J, Megden T, Padfield PL, Parati G, O’Brien E; Working Group on Blood Pressure Monitoring of the European Society of Hypertension. Self monitoring of blood pressure at home. BMJ. 2004;329(7459):870-1.

10. Pierin AMG, Costa KRA, Gusmão JL, Caetano El, Ortega K, Mion Juniorr $D$. $O$ efeito benéfico da medida residencial da pressão arterial (MRPA) na avaliação do controle da hipertensão arterial. Hipertensão. 2007;10(2):62-5.

11. Mancia G, Parati G, Pomidossi G. Alerting reaction and rise in blood pressure during measurement by physician and nurse. Hypertension. 1987;9(6):209-15.

12. Batide-Alanore A, Chatellier G, Bobrie G, Fofol I, Plouin PF. Comparison of nurse and physician determined clinic blood pressure levels in patients referred to a hypertension clinic: complications for subsequent management. J Hypertens. 2000;18(4):391-8.

13. Pickering TG. Measurement of blood pressure in and out of the office. J Clin Hypertens. 2005;7(2):123-9.

14. Pierin AMG, Gusmão JL, Mano GVF, Ignes EC, Ortega K, Mion Jr D. White coat hypertension and white coat effect: blood pressure measurement taken by patient, by the nurse, by the physician, and routine measurement in hypertensive outpatients. Hipertensão. 2008;11(3):98-104.

15. Campbell NRC, Milkovich L, Burguess E, Mckay DW. Self-measurement of blood pressure: accuracy, patient preparation for readings, technique and equipament. Blood Press Monit. 2001;6(2):133-8.
16. Pierin AMG, Ignez EC, Jacob Filho W, Barbato AJG, Mion Junior D. Blood pressure measurements taken by patients are similar to home and ambulatory blood pressure measurements. Clinics (São Paulo). 2008;63(1):43-50.

17. Segre CA, Ueno RK, Karim RJ, Warde TA, Accorsi MH, Miname $\mathrm{R}$, et al. White-coat Hypertension and Normotension in the League of Hypertension of the Hospital das Clínicas, FMUSP: prevalence, clinical and demographic characteristics. Arq Bras Cardiol. 2003;80(2):122-6.

18. Ayman D, Goldshine AD. Blood pressure determinations by patients with essential hypertension: the difference between clinic and home readings before treatment. Am J Med Sci. 1940;200:465-74.

19. Zhu N, Bu M, Chen D, Li T, Qian J, Yu Q, et al. A study of the white-coat phenomenon in patients with primary hypertension. Hypertens Res. 2008;31(1):37-41.

20. Parati G, Omboni S, Albini F, Piantoni L, Giuliano A, Revera $M$, et al. Home blood pressure telemonitoring improves hypertension control in general practice. The TeleBPCare study. J Hypertens. 2009;27(1):198-203.

21. Agena F, Silva GCA, Pierin AMG. Home blood pressure monitoring: updates and the nurse's role. Rev Esc Enferm USP [Internet]. 2011 [cited 2011 May 17];45(1):258-63. Available from: http://www.scielo.br/pdf/reeusp/v45n1/en_36.pdf 